In Crescendo. Institucional. 2016; 7(2): 63-71

Fecha de recepción: 29 de abril de 2016

Fecha de aceptación: 9 de noviembre de 2016

\title{
CONTROL Y VALORACIÓN PARA LA ZONA DE DESARROLLO PRÓXIMO EN MÉXICO
}

\section{CONTROL AND ASSESSMENT FOR THE ZONE OF PROXIMAL DEVElopment IN MeXICO}

\author{
José Manuel Castorena Machuca* \\ Patricia Castorena Briones**
}

\begin{abstract}
RESUMEN
$\mathrm{E}_{\mathrm{en}}^{\mathrm{s}}$ s muy importante para el maestro propiciar la autorregulación del sujeto o alumno, en su actividad de aprendizaje, porque así se eleva la calidad del proceso de enseñanza-aprendizaje y se potencia el desarrollo cognitivo individual, de acuerdo con sus etapas de desarrollo. Hay que asegurarse de que se vaya produciendo una asimilación productiva y consciente del contenido, utilizando una metodología de control y valoración de las actividades de aprendizaje, lo cual se hace mediante una comparación de tareas entre pares y dirigiendo que tengan un grado de correspondencia con el modelo original. Esto genera que los alumnos sean más exigentes con sus actividades de aprendizaje y los lleve a mejores niveles de eficiencia mediante el potenciamiento de la zona de desarrollo próximo de los educandos. Ese análisis crítico de tareas por parte de los alumnos tiene un mayor impacto si se hace de manera anónima para que la emotividad entre pares no desvíe el objetivo que se pretende. Es fundamental para crear conciencia en los alumnos, la importancia de estar centrados en cuanto a su psicología del aprendizaje y que la apliquen en elevar la calidad de su aprendizaje conforme avanzan en sus diferentes niveles educativos o que ya como egresados en su campo laboral para que internalicen que no solo fue pasar el tiempo, sino que hicieron suyo el contenido que les propusieron los profesores, mediante una participación colaborativa y de valoración para llegar a ser más autónomos mentalmente en su desarrollo cognitivo (Constance Kamii).
\end{abstract}

Palabras clave: valoración, zona de desarrollo próximo, pedagogía, aprendizaje significativo, implementación, evaluación

* Catedrático de Ingeniería Industrial en el Instituto Tecnológico de San Luis Potosí. Correo electrónico: jmktatcslp01@yahoo.com.

** Universidad del Valle, San Luis Potosí. Correo electrónico: jmkatate21@gmail.com. 


\section{ABSTRACT}

It is very important for the teacher encourage self-regulation of the subject or student in his learning activity, because that raises the quality of the teaching process-learning. So that individual cognitive development, according to their developmental stages is enhanced. Make sure you go producing a productive assimilation and aware of the content, using a control methodology and assessment of learning activities, which is done by comparing tasks among peers and directing that have a degree of correspondence with the original model, which causes students to be more demanding of their learning activities and leads to higher levels of efficiency by enhancing be ZPD of students. That critical task analysis by students has a greater impact if done anonymously so that emotionality peer does not divert the objective sought. It is essential to raise awareness in the student, the importance of being focused in their psychology of learning and apply it to raise the quality of their learning as they progress in their different educational levels or already as graduates in their field to internalize that was not only spend time but endorsed the content that proposed them teachers, through collaborative participation and valuation to become more autonomous in their cognitive development mentally (Kamii).

Keywords: assessment, development, zone of proximal development, pedagogy, meaningful learning, implementation, evaluation

\section{INTRODUCCIÓN}

Se considera que el control y la valoración (Tovar Gutiérrez, 2015) tienen gran relevancia para potenciar la zona de desarrollo próximo (Vygotsky, 2020). Desde el punto de vista administrativo de un curso que imparta un maestro, el control es vigilar que se cumpla lo planeado, y si hay alguna ligera desviación, tomar las medidas necesarias para llevar las actividades alineadas con el objetivo para poder alcanzarlo.

Y la valoración se refiere a una metodología aplicada como control a los alumnos para que vayan construyendo su conocimiento, en base a sus conocimientos previos o sus bases socioculturales para que hagan de los nuevos conocimientos que se les proponen y que potencian su zona de desarrollo cognitivo hasta llevarlos a un grado de autonomía mayor.

Se le llama valoración porque son los mismos alumnos lo que valoran las actividades de aprendizaje de sus compañeros y viceversa, lo cual el maestro va controlando en grados de avance para que logren la madurez cognitiva y mediante la motivación de profesor participen activamente y de manera proactiva en ir mejorando sus tareas al reconocer el trabajo de sus compañeros y que se dé un ambiente amigable en el salón de clases y de crear el interés por aprender y la confianza por parte del profesor, entre los equipo de trabajo para resolver problemas dentro del aula y que tengan que ver con sus contextos sociales. 


\section{Plataforma teórica}

- Lo biológico y lo sociocultural en el desarrollo del ser humano. Se decía que el ser humano nace como "una tabula rasa" (Aristóteles, 300 a. C.). En este artículo se considera que ya no es así, porque se ha comprobado que el ser humano, ya en el vientre materno, es capaz de distinguir las voces de diferentes familiares y reaccionar de manera favorable o con disgusto según sea el caso y la manera en que se le estimule, de tal manera que siente, ve y oye desde la semana 22 (Hoyos y Puccini, 2015). De la misma manera, si el no nato es estimulado con música clásica, por ejemplo, con lecturas que estimulen su lenguaje, cuando nazca ese nuevo ser vendrá con toda una serie de potencialidades, para tener un oído musical o para tener gusto por la lectura. El medioambiente biológico también influirá para la buena o mala salud. Así, dependiendo de los grados de contaminación del aire, del agua, del mar, de los ríos, de los alimentos, en fin, de todos los seres bióticos y abióticos, se puede provocar un descenso de la calidad de vida y su promedio de vida. O, por el contrario, si el medioambiente es sano, el ser humano crecerá sano y tendrá mejores oportunidades de alcanzar un mejor ciclo de vida y calidad.

En cuanto a lo social y lo cultural, desde luego que influye pues a priori no es lo mismo la música clásica y lecturas de reconocido bagaje cultural, que otra clase de música y pasquines de dudosa calidad cultural que le lean al ser en formación.

Sin embargo, no hay que olvidar que los seres humanos son complejos y que la perspectiva histórico-cultural ante la relación biología-cultura (Greenfield y Bruner, 1966) y el rol tan importante que juegan las experiencias de vida en los educandos conforme avanzan en sus grados académicos.

De tal forma, pues, hay que decir que las experiencias negativas o la ausencia de la estimulación adecuada en el desarrollo temprano de los seres humanos pueden tener consecuencias serias y sostenidas a lo largo de la vida educativa.

No se puede negar que el maltrato, la negligencia y los problemas asociados con la desigualdad social impactarán adversamente el desarrollo mental de los educandos. Esto generaría condiciones que constituyen factores de riesgo en todas las dimensiones socioculturales y del desarrollo, ya que ponen de manifiesto la vulnerabilidad social del cerebro ante conocimientos más complejos que se les proponen ser asimilados. Y estarían en desventaja con educandos que sí tienen los medios para salvar esos obstáculos, y no solo se refiere a medios materiales, sino también de afecto y necesidades espirituales.

Cabe mencionar nuevamente que la atención biológica en la salud de los niños estará estimulada por la buena educación que reciban en sus hogares y, más tarde, por medio de la educación en las escuelas.

Y son precisamente los estados de ánimo y el trato de las personas que cuidan al niño (padres de familia y pedagogos) los que son determinantes en su desarrollo intelectual y afectivo, así como en su conducta en la vida futura conforme vayan avanzando en sus niveles educativos y sociales. 
Cabe mencionar aquí que todos los seres humanos pueden manejar cierto nivel de estrés que estimule la voluntad para terminar con las tareas asignadas o los retos que les propongan; pero un exceso de situaciones estresantes durante la primera infancia puede formar un patrón en la manera en que los niños reaccionan ante el estrés en años posteriores (Cichetti y Walker, 2001).

\section{- Diagnóstico pedagógico}

El maestro, por lo general, parte de este diagnóstico para planear las actividades de aprendizaje de los alumnos, pues tiene que distinguir que no todos los alumnos llegan con el mismo nivel de aprendizaje; de tal manera que si desea seguir un aprendizaje personalizado, estará controlando el avance cognitivo, sabiendo que todos los alumnos son diferentes, sea en edad, sexo, cultura, extracción social, gustos y que aprenden de diferente manera, pues unos son quinestésicos, visuales y auditivos y todavía son diferentes en sus inteligencias múltiples (Gardner,1983).

Así que, es a partir de ese diagnóstico escolar que el profesor estará en posibilidad de ir dosificando el conocimiento o el contenido de acuerdo con las capacidades individuales de sus alumnos. Y sin olvidar que todos los alumnos tienen sueños diferentes por los que deciden estudiar y que los profesores no deben destruir esos sueños, sino proponerles contenidos edificantes o significativos que los ayuden a lograr más rápido esos sueños.

Un doctor de alguna especialidad médica realiza un diagnóstico para poder enfrentar el problema de salud que padece el paciente, por lo que le hace algunas preguntas y, con base a esas respuestas maquilladas o no por el mismo paciente, el médico con su experiencia y conocimiento conducirá al enfermo a recobrar su salud mediante la receta de la medicina certera de la aplicación de su juicio científico.

De manera análoga el maestro debe hacer un diagnóstico a sus alumnos para ver qué tanto saben de los requisitos para estar en su nivel educativo donde se inscribieron y del contenido que les va a ofrecer para establecer un plan y una estrategia didáctica que le permitan llevar al educando a mejores niveles cognitivos, y donde el profesor aplicará su juicio pedagógico en la ciencia de la educación.

\section{- Teoría de Vygotsky sobre la zona del desarrollo próximo (ZDP)}

Este pedagogo sostiene que el desarrollo tecnológico en los estudiantes debe ser entendido como una serie de transformaciones cualitativas simbólicas como lo es el lenguaje y cualitativas concretas, como lo son las interacciones socioculturales con sus compañeros y con el ambiente social en su comunidad, donde lo más conveniente es que asimile y conozca y comprenda la cultura que lo rodea mediante una relación que llama interindividual. Y que cuando los maestros estimulan su zona de desarrollo próximo por medio de instrumentos socioculturales, pasan de una zona real a una de desarrollo potencial. Esta guía, que se da por medio de un "andamiaje" que sitúa al profesor como un facilitador en la construcción del 
aprendizaje de los alumnos, puede lograr un nivel más complejo del contenido que le propone y que le presenta como deseable de alcanzar, el cual debe ser retórico y real de lograr, lo que les da más autonomía mental mediante una relación denominada interindividual, donde al ser estimulados sus centros de interés por el aprendizaje, se construye su desarrollo cognitivo en un nivel superior y así sucesivamente, pasando por los diferentes niveles educativos en una dialéctica dinámica de construcción del conocimiento y hasta presentar una forma madura y autónoma para la resolución de problemas que mejoren la sociedad con la aplicación de sus funciones psicológicas superiores.

\section{- Madurez y pensamiento}

Este concepto tiene también su base en Vygotsky, el cual dice que, definitivamente, para un nivel superior de desarrollo cognitivo, influye con determinación el contexto sociocultural.

Y a su vez, la cultura proporciona herramientas para modificar el entorno físico y social para las siguientes generaciones de estudiantes, en una espiral dialéctica de mejora continua. El desarrollo de las sociedades se origina a partir de las relaciones sociales y la cultura, lo que va transformando la sociedad a ser mejores en todos los aspectos (ideal).

Los profesores promueven el desarrollo de todas las aptitudes de que son susceptibles sus estudiantes, desarrollando sus competencias hasta alcanzar la habilidad rápida, certera y madura de aplicación de lo que puede ser una aptitud innata o desarrollada que trae el educando de un cierto nivel educativo y aterrizar esa habilidad en la práctica, el entrenamiento y crecer en la experiencia que permite que la persona logre mejorar sus habilidades y sus procesos para lograrlos, y que con el paso del tiempo, inclusive, adquirir nuevas habilidades en una espiral constante de construcción de conocimientos.

Tomando en cuenta que la formación de una habilidad comprende una etapa en la adquisición de conocimientos de los modos de actuar socio culturalmente, y que cuando bajo la dirección del profesor o algún experto que utiliza un andamiaje que le facilite el aprendizaje. De tal forma que el estudiante o aprendiz, reciba la orientación adecuada sobre la forma de proceder, actuar para lograr el mejoramiento de su habilidad requerida para el siguiente nivel educativo o ya para el campo laboral y social.

Entonces se puede argumentar que un desarrollo cognitivo superior normalmente está dirigido como decía Platón en sus Diálogos, hacia el bien, la justicia y la belleza.

Aplican la sensatez, el buen juicio y la prudencia cuando toman decisiones en todos los proyectos ya sean personales o grupales. Y se les conoce como personas auto realizadas y con una elevada posesión espiritual que exige compromiso de transformar el mundo social a algo mejor con una cosmovisión de no extinguir 
a las especies, ni poner en riesgo la pripia extinción de la especie humana al no producir bienes y servicios sin aplicación de un desarrollo sostenible con todos los seres bióticos y abióticos.

La etapa de la madurez, es la maravillosa estadía en la que el ser humano alcanza su momento de máximo desarrollo físico, psíquico, intelectual y emocional, logrando tener un equilibrio en su persona e incluso un control en ella misma. Es un proceso de mejoramiento constante, y todavía perfectible, y lo que a la vez está ligado a otro proceso de independencia mental. Por lo que no solo es una acumulación de años, sino de conocimientos, de sabiduría, de equilibrio físico y emocional.

El crecimiento mental maduro, en realidad es un proceso de desarrollo integral, que cada persona puede experimentar de manera continua, ya sea en los diferentes niveles educativos o sea maduro en su nivel y así durante toda su existencia. No es una acción que se logre de un momento a otro o buscando atajos, sino que más bien, es el resultado de un largo proceso que se va manteniendo en el tiempo, creando espacios y construyendo circunstancias más favorables, que permiten al individuo el ir aumentando continuamente sus conocimientos, sus capacidades, sus habilidades, su potencial y, en fin, todo su desarrollo emocional, biológico, espiritual y en todos los aspectos personales y factores influyentes en la vida de cada persona.

El lector puede intuir que el desarrollo humano consiste en la libertad, que a su vez respeta el derecho de otras personas y la formación y expansión de las capacidades humanas (competencias), es decir, en la ampliación de la gama de cosas, opciones y oportunidades que las personas pueden hacer, llegar a ser, tener y convivir armoniosamente con su entorno social y cultural.

\section{DESARROLLO}

Se propone en este artículo que el profesor trate de involucrar a los alumnos en el proceso de corrección de sus actividades de aprendizaje entre pares para que, por un lado, el maestro logre el control sobre estas; pero si los alumnos hacen una valoración de esos aprendizajes los harán más independientes en su avance cognitivo y ya no verán solo al maestro como un ogro que los califica o los descalifica.

De tal forma que el profesor parte de la zona real de desarrollo que tienen sus estudiantes, les dice el objetivo del conocimiento nuevo que les propone, y mediante esa valoración logra que los estudiantes tengan un conocimiento mayor, estimulando la zona de desarrollo próximo de los estudiantes; y una vez alcanzado el objetivo, este será la nueva zona real y se les propondrá otro nivel de conocimiento y así sucesivamente. Así, los estudiantes estarán construyendo sus propios conocimientos y estarán avanzando socioculturalmente hacia cada nivel educativo hasta que se inserten finalmente en el campo laboral e intenten mejorar su entorno social y ecológico. 
El maestro estará pendiente de los grados de esa valoración, guiando a los alumnos o haciendo que participen en las reglas de cómo se llevará entre ellos esa valoración o evaluación entre pares, respetando a sus compañeros.

Al trabajar de esa manera, los alumnos estarán construyendo su calidad de educación, ya que lograrán mejores niveles de correspondencia entre lo que se debía haber hecho, ya sea lo realizado individualmente o por el consenso grupal.

Se considera que lo anterior les dará a los alumnos un juicio más crítico para evaluar el trabajo de sus compañeros y con la retroalimentación de sus pares, mejorará también el suyo.

En un ejemplo universitario, se divide al grupo de alumnos en equipo de tres o cuatro alumnos. Si el examen de conocimiento vale 6 puntos; tareas, 3 puntos; asistencia, 1, la calificación para el alumno será de 10 si cubre completamente los rubros.

Para el control de las actividades de aprendizaje, el maestro planea los puntos relevantes que calificarán la tarea, por ejemplo, un resumen sobre un tema de alguna de las unidades a evaluar. La valoración consiste en que lo alumnos se evalúen en pares el resumen, ya sea que lo hagan por medio de alguna presentación digital o como acuerden los mismos alumnos, lo que sin duda tiene ventajas para el profesor, pues así no solo lo verán como el ogro que examina, sino que los educandos también participarán en la construcción de sus conocimientos, logrando mejores niveles de desarrollo cognitivo y autonomía mental. Además, la evaluación del curso deber ser integral, abierta y transparente para los agentes que intervienen en el proceso enseñanza-aprendizaje.

El control y la valoración se aplican en el nivel preescolar, cuando los alumnos presentan una exposición de sus trabajos de pintura o de plastilina, donde plasman sus competencias artísticas y que los lleva a potenciar su zona de desarrollo próximo, lo que a su vez les va dando autonomía mental.

\section{CONCLUSIÓN}

Se puede inferir en este artículo por parte de los profesores que las ideas sobre el desarrollo humano por medio de la aplicación de las teorías pedagógicas juegan un papel muy importante y que impactan sobre las prácticas educativas que hagan los maestros en su nivel de influencia dentro del aula. Esas prácticas educativas pueden transformar las ideas sobre el desarrollo humano si se mejoran el control y la valoración entre pares por parte de los educandos. La elección inteligente es optar por dichas prácticas educativas que permitan avanzar a la zona de desarrollo próximo, y conducirán a los maestros una mejor calidad de la educación para ellos mismos y para sus alumnos, ya que estarán propiciando ambiente amigables de aprendizaje donde los alumnos participen en forma dinámica en la valoración individual y grupal.

La estimulación por parte de los maestros hacia sus alumnos, despertando los puntos de interés en sus propios aprendizajes hará que crezcan cognitivamente y logren mejores grados de madurez mental y cognitiva. 
Se puede decir que los alumnos, mediante ese adecuado o andamiaje construido por el profesor alrededor de las unidades de aprendizaje de los alumnos en cada unidad y de acuerdo al nivel educativo de que se trate, propiciará que sus alumnos construyan sus propios conocimientos que los haga más hábiles para competir en su siguiente nivel educativo o ya para su vida social o laboral, pues habrá alcanzado un grado de madurez física, emocional y mental que los libera como verdaderos seres humanos, con autonomía mental para tomar sus propias decisiones, respetando la libertad de los demás y promoviendo la no extinción de las especies vivas. El docente está mejorando así la calidad vida de los estudiantes mediante la poderosa herramienta que es la educación logrando un mejor nivel cognitivo en los alumnos para enfrentar la incertidumbre con decisiones rápidas, pero certeras.

\section{REFERENCIAS BIBLIOGRÁFICAS}

Báxter Pérez, Esther. (2002). ¿Cuándo y cómo educar en valores? Cuba: Instituto Central de Ciencias Pedagógicas.

Bruner, J. S. (1972). Hacia una teoría de la instrucción. Edición Revolucionaria.

Castorena Machuca, J. M. (1996). La tecnología educativa aplicada en el proceso de la ensenanza y el aprendizaje. Cuba: ITSLP.

Corral Ruso, R. (2001). El concepto de zona de desarrollo próximo: una interpretación. La Habana: Universidad de la Habana.

Davidov, V. V. y Markova A. K. (1987). La concepción de la actividad de estudio de los escolares en la psicología evolutiva y pedagógica en la URSS. Moscú: Editorial Progreso.

Elkonin, D. B. (1986). Las cuestiones psicológicas relativas a la formación de la actividad docente en la edad escolar menor. Lliasov y V. Ya. Limüdis. Antología de la psicología pedagógica y de las edades. La Habana: Editorial Pueblo y Educación.

Garbanzo Vargas, G. (2007). Factores asociados al rendimiento académico en estudiantes universitarios. Costa Rica: Universidad de Costa Rica.

Galperin, P. Ya (1981). Sobre el problema de la atención. V. V. Mirenenko y A. V. Petrovsky. Antología de Psicología. Cuba: Editorial Prosveshemie.

Kamii, C. (2013). La autonomía como finalidad de la educación. Illinios: Universidad de Illinois.

Lipquina. A. I. (1976). Cómo determinar la autovaloración del escolar. Revista Znanie, 12. Serie Pedagogía y Psicología.

López, J. y otros. (1979). Algunos aspectos de la dirección pedagógica de la actividad cognoscitiva de los escolares. MINED. Documentos normativos y metodológicos (primera parte).

Rico, P. (1987), ¿Cómo el alumno evalúa los resultados de sus tareas docentes? Temas de Psicología Pedagógica para maestros. La Habana: Editorial Pueblo y Educación. 
Rico, P. (1998). La formación de acciones de control y valoración de actividades de aprendizaje en escolares primarios. Cuba: Instituto Central de Ciencias Pedagógicas.

Roloff, G. (1983). Particularidades del desarrollo de la autovaloración en escolares primarios. Revista Ciencias Pedagógicas, 6. Año IV, enero-junio.

Talizina, N. F. (1987). La formación de la actividad cognoscitiva de los escolares. La Habana: Universidad de La Habana-Ministerio de Educación Superior.

Wallon, H. (1946). El rol del otro en la conciencia del yo. Selección de lecturas sobre psicología de la personalidad. La Habana: Universidad de La Habana. 